\title{
A hybrid predictive technique for lossless image compression
}

\author{
N. A. N. Azman ${ }^{1}$, Samura Ali ${ }^{2}$, Rozeha A. Rashid ${ }^{3}$, Faiz Asraf Saparudin ${ }^{4}$, Mohd Adib Sarijari \\ 1,2,3,4 Universiti Teknologi Malaysia, Malaysia \\ ${ }^{1}$ Universiti Tun Hussein Onn Malaysia, Malaysia
}

\begin{tabular}{l}
\hline \hline Article Info \\
\hline Article history: \\
Received Mar 29, 2019 \\
Revised May 31, 2019 \\
Accepted Jul 9, 2019 \\
\hline
\end{tabular}

Keywords:

Differential pulse code

Modulation

Huffman coding

Image compression

Integer Wavelet Transform

Wireless communication

\begin{abstract}
Compression of images is of great interest in applications where efficiency with respect to data storage or transmission bandwidth is sought.The rapid growth of social media and digital networks have given rise to huge amount of image data being accessed and exchanged daily. However, the larger the image size, the longer it takes to transmit and archive. In other words, high quality images require huge amount of transmission bandwidth and storage space. Suitable image compression can help in reducing the image size and improving transmission speed. Lossless image compression is especially crucial in fields such as remote sensing healthcare network, security and military applications as the quality of images needs to be maintained to avoid any errors during analysis or diagnosis. In this paper, a hybrid prediction lossless image compression algorithm is proposed to address these issues. The algorithm is achieved by combining predictive Differential Pulse Code Modulation (DPCM) and Integer Wavelet Transform (IWT). Entropy and compression ratio calculation are used to analyze the performance of the designed coding. The analysis shows that the best hybrid predictive algorithm is the sequence of DPCM-IWT-Huffman which has bits sizes reduced by $36 \%, 48 \%, 34 \%$ and $13 \%$ for tested images of Lena, Cameraman, Pepper and Baboon, respectively.
\end{abstract}

Copyright () 2019 Institute of Advanced Engineering and Science. All rights reserved.

\section{Corresponding Author:}

Samura Ali,

School of Electrical Engineering, Universiti Teknologi Malaysia, 81310 Johor Bahru, Johor, Malaysia. Email: samurah@utm.my

\section{INTRODUCTION}

In today's modern era, multimedia communication has become a norm and gives a huge impact on human's lives. There is a tremendous amount of image data being shared and accessed on social media networks such as Facebook, Twitter and Instagram. In addition, due to the Industry Revolution 4.0 and Internet-of-Things (IoT) technology, the trend of exchanging and controlling almost everything wirelessly is likely to continue far into the future. Even though storage costs are decreasing, the volume of digital image data to be transmitted and stored is increasing at a much faster rate. It is also more susceptible to noise during transmission. Image compression technique can be deployed in order to promote faster transmission speed and reliable data transmission. However, quality images are very crucial for fields like military applications, healthcare and biometric-based security solutions using fingerprint or face recognition. Due to its noiseless characteristic, lossless image compression is beneficial when it comes to handling image that must maintain its quality and original form.

High compression ratio and the ability to decode the compressed image's data at various resolutions is necessary for a compression process [1]. The image is compressed by reducing the spatial and spectral redundancy so that the image can be stored and transmitted in a proper manner [2]. By performing image compression, a large size of image data can be managed and stored efficiently with the use of suitable 
technique. Image compression can increase the amount of the storage space by encoding the original image with limited bits. The transmission time to upload a compressed image on the internet and get a download from the webpages becomes shorter. If there is a loss of data during compression, it will lead to the problem during analyzing the data as the resulted analysis is not precise. Therefore, there is a need to develop a good image compression technique that is almost error free and can produce a reconstructed image that as true to its original form as possible.

There is a lot of research works that has been conducted in lossless image compression. Some of the common lossless image compression are LZW, DEFLATE, LOCO-I, JPEG-LS, run-length encoding JPEG, JPEG 2000 and etc [3-25]. According to Amira Mofreh et.al, combining Linear Predictive Coding with Discrete Wavelet Transform and-Huffman (LPCDH) gives faster and higher compression ratio compared to DWT-Huffman [22]. C Jain, V Chaudhary, K Jain et.al has proposed a different method based on lossless image compression where it uses Wavelet Packet Transform (WPT) followed by lifting scheme [23]. Based on their analysis, Integer Wavelet Packet Transform (IWPT) speed up the decomposition process and gives high compression ratio compared to WPT. The other proposed method of lossless image compression is performed by Vineeta G. A.G. Rao and K. Mohan Pandey where they proposed Integer wavelet transform as a predictive method [25]. The resulted studies show that they achieved maximum compression ratio when the coefficient is added and varies in lifting scheme. The proposed method proves that predictive Integer wavelet transforms with coefficient gives high compression ratio compared to Integer Wavelet Transform (IWT) without coefficient.

This paper focuses on developing an algorithm for hybrid predictive coding by combining predictive Differential Pulse Code Modulation (DPCM) and IWT. The performance of DPCM-IWT and IWT-DPCM is compared and the best hybrid predictive coding will be chosen to be combined with Huffman entropy coding for further compression process. Several tables of truncated Huffman have been designed in order to achieve maximum compression ratio. Five grayscale images have been used to test the compression engine. The image compression engine is done by designing the MATLAB code by using MATLAB software model. The remainder of this paper is organized as follows. The background and the methodology of the lossless compression techniques are presented in Section 2. Then, Section 3 discusses the results. Lastly, the conclusion is drawn in Section 4.

\section{LOSSLESS IMAGE COMPRESSION TECHNIQUES}

Traditional transform-based methods for compression, while effective, are lossy. In certain applications, such as biomedical image analysis, even slight compression losses can be unacceptable. Therefore recent research has focused on finding more efficient ways of achieveing lossless compression; i.e., the process of compressing images without any degradation in quality or any quantization losses. This section discusses the methodology that is used to achieve lossless image compression. Basically, the proposed lossless image compression is constructed by combining predictive coding and entropy coding. As for predictive coding, it is performed by combining two types of predictive coding which are IWT and DPCM where the pixels are predicted through these predictive codings. After the pixel is predicted, the image is further compressed with entropy coding.

The basic step and flowchart for the proposed work are given as follows. The first step is to read the image. Next, DPCM encoder is performed where the pixels are read by column and row and predict the pixel. DPCM exploits the inter-plane correlation by producing a difference matrix with an average value much smaller than the average value of the original image. It reduces the number of binary $1 \mathrm{~s}$ in the more significant bit planes, and therefore increases compression because run-length encoding will have fewer groups of binary $1 \mathrm{~s}$ to encode. This is followed by IWT encoder by applying lifting scheme and Haar wavelet transform. The output of IWT will be converted to positive integer. Next step is to calculate the frequency occurrence symbol and calculate the entropy. Then, the pixel distribution is computed followed by a display of the distribution graph of hybrid predictive coding.

The positive integer is then converted into 1-dimension (1D) before Huffman compression is performed based on the designed Huffman truncated table. Next, the entropy value of the compression images is computed based on the resulted codeword. Lastly, the compressed codeword is decoded in order to recover the original images. Figure 1 shows the block diagram of the proposed lossless image compression. 


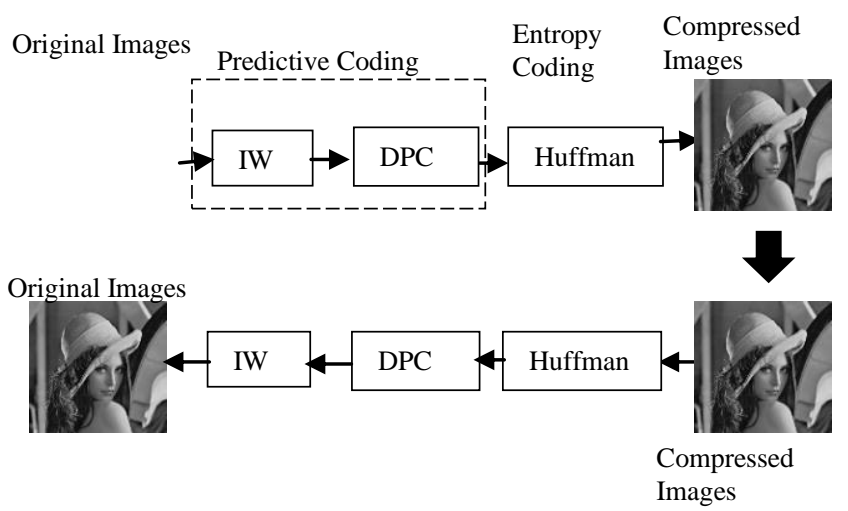

Figure 1. Block diagram of the proposed lossless image compression

\subsection{Different Pulse Code Modulations (DPCM)}

DPCM is one of the proposed predictive coding techniques. DPCM prediction is nonlinear. Its basic idea is to predict the information in every image pixel and make the predicted image entropy less than the original image entropy. Because there is strong correlation among adjacent pixels, current pixel values are predicted by pixel knowledge. It works by predicting the pixels value where the pixel error will be the resulted output. Pixel error is obtained by subtracting the original pixel with the predicted pixel value. The resulted pixel error will have a lower zero-order and concentrated near to zero. The DPCM algorithm was designed by using the formula in (1).

$$
\operatorname{PixErr}_{(x, y)}=\operatorname{Im}_{(x, y)}-\left[\mathrm{k} 1\left(\operatorname{Im}_{(x-1, y)}\right)+\mathrm{k} 2\left(\operatorname{Im}_{(x, y-1)}\right)-\mathrm{k} 3\left(\operatorname{Im}_{(x,-1 y-1)}\right)\right]
$$

Figure 2 and 3 depict the original pixel and the obtained pixel error, respectively.

$\begin{array}{llllllll}96 & 97 & 95 & 97 & 102 & 106 & 107 & 108 \\ 123 & 99 & 97 & 100 & 109 & 117 & 120 & 122 \\ 103 & 104 & 110 & 116 & 118 & 117 & 116 & 117 \\ 109 & 108 & 110 & 108 & 102 & 105 & 118 & 133 \\ 109 & 103 & 108 & 109 & 112 & 125 & 140 & 143 \\ 100 & 113 & 115 & 129 & 136 & 131 & 141 & 146 \\ 114 & 103 & 110 & 129 & 139 & 132 & 139 & 149 \\ 112 & 94 & 90 & 99 & 115 & 123 & 134 & 141\end{array}$

Figure 2. Original pixel value

$\begin{array}{llllllll}0 & 1 & -2 & 2 & 5 & 4 & 1 & 1 \\ 27 & -11 & -1 & 2 & 7 & 10 & 8 & 8 \\ -8 & 4 & 9 & 9 & 4 & -2 & -3 & -2 \\ 6 & 0 & -1 & -6 & -11 & -5 & 8 & 16 \\ 1 & -6 & 2 & 3 & 6 & 14 & 15 & 7 \\ -6 & 10 & 4 & 16 & 12 & -4 & 5 & 4 \\ 8 & -11 & -3 & 8 & 8 & -6 & 2 & 7 \\ 4 & -15 & -17 & -13 & -2 & -3 & 1 & -1\end{array}$

Figure 3. Pixel error

\subsection{Integer Wavelet Transform (IWT)}

IWT is the other predictive coding that is used in this proposed method. By performing IWT, it can map integer to integer where it requires shorter data length compared to floating point. Integer wavelet transforms was developed by Win Sweldens where it was designed based on lifting scheme [23].

Lifting scheme allows IWT to compute faster since it produces integer number. By performing IWT, it allows performing reversible image compression [23]. Lifting scheme is done by applying Haar wavelet 
transform as mother wavelet because it is easy to compute and simple. IWT is constructed based on three basic steps which are spilt, predict and update [23-25].

By applying lifting scheme into IWT, it will result in image decomposition where the image is sub-sampled into parts where the resolution is decreased from one level to the next level until the desired resolution is achieved as shown in Figure 4 [23].

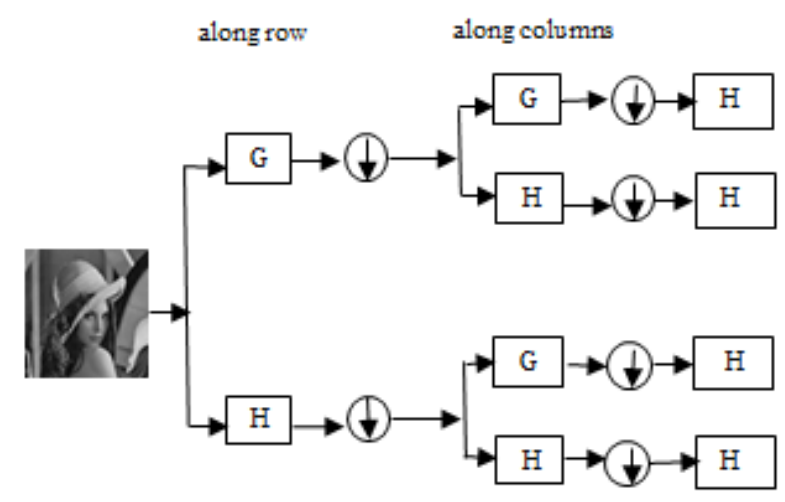

Figure 4. Image decomposition by applying lifting scheme

The image is filtered by using high pass filter $(\mathrm{G})$ and low pass filter $(\mathrm{H})$. It starts by filtering the row parts followed by the column parts. The images are then down-sampled by 2 to produce high and low-frequency components. Different sub-bands are produced during IWT encoding where it consists of one approximation and three details where LL (approximation sub-band), HL (horizontal sub-band), LH (vertical sub-band) and $\mathrm{HH}$ (diagonal sub-band) are resulted from the first level decomposition and second stage of filtering. This is shown in Figure 5.

\begin{tabular}{|c|c|c|c|}
\hline LL & HLn & \multirow{2}{*}{ HL2 } & \\
\cline { 1 - 1 } LHn & HHn & HL1 \\
\hline LH2 & HH2 & \\
\hline & & \\
& LH2 & HH2 \\
& & \\
\hline
\end{tabular}

Figure 5. Multiresolution scheme after several levels of wavelet

\subsection{Entropy coding}

Entropy coding is used as a compression part in lossless image compression. Entropy coding or run-length encoding produces compression by replacing a sequence of binary data with a sequence of hexadecimal numbers, each of which represents the number of occurrences of the same binary value in an unbroken string. The simplest entropy coding is a static Huffman truncated table. Although dynamic Huffman coding will produce better compression than static coding (because the dynamic code is specially customized to the data), this superior performance is impacted severely by the need to include the code key as a table along with the header data. Because there is the almost certain probability that the code key will be different for every bit plane and every image block, the header penalty would be too great.

A Huffman truncated table has been constructed where it is consists of tail and head where it was designed based on the pixels frequency occurrence produced from the hybrid predictive coding. The truncated Huffman coding can cover up to 512 number of symbols. The output of predictive coding will be converted to the positive integer and then will be compressed by Huffman truncated coding. A codeword is 
produced after compression process where head and tail are concatenated together. The pixels that have the highest probability will be assigned to the shortest codeword based on the generated pixel distribution graph. The produced codeword contains lesser bits compared to the original bits.

\section{RESULTS AND ANALYSIS}

The results are obtained from the implementation of the proposed method as provided on the block diagram in section 3 . The performance of the proposed hybrid predictive method was analyzed by conducting image compression by using two different methods. For the first method, hybrid predictive IWT-DPCM was performed. For the second method, hybrid predictive DPCM-IWT was performed on the same image and the resulted compression ratio is calculated on both methods to observe which method is the best hybrid predictive algorithm. The input image that is used for testing is in BMP format and the image resolution is $512 \times 512$. The designed algorithm has been tested on a set of 5 grey images with the same format and size as shown in Figure 6. This test image is a well known standard test image used by most established methods. Since current image sensors use higher resolution, this result for 512x512 image will be useful to satisfy the higher resolution image requirement.

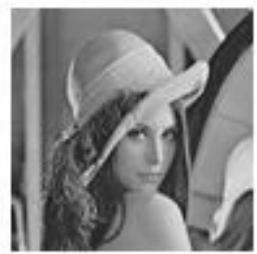

(a)

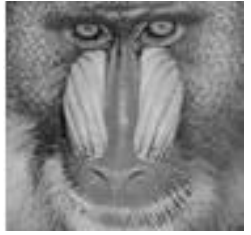

(b)

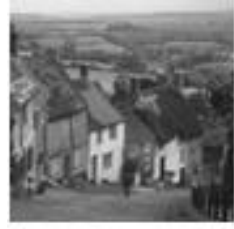

(c)

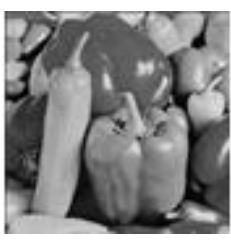

(d)

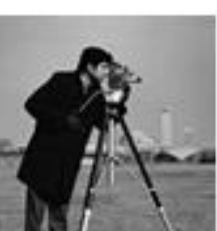

(e)

Figure 6. Original images, (a) Lena, (b) Baboon, (c) Goldhill, (d) Peppers, (e) Cameraman

Table 1 shows the comparison of entropy for the two methods which are IWT-DPCM and DPCM-IWT, respectively. Most established methods didn't provide entropy for predictor. Main objective from this paper is to find the best combination between DPCM and IWT. Based on the table, the entropy value of method 2 is lesser than method 1 . The cut-off pixel distribution graph that has been calculated in every picture shows that most of the image is having a high probability of pixels around the range of -50 to 50. Therefore, less codeword of Huffman truncated table is assigned to the pixels in between the range of -50 and 50 to achieve maximum compression ratio.

Table 1. Entropy comparison

\begin{tabular}{ccc}
\hline Images & \multicolumn{2}{c}{ Predictive method } \\
& Method 1 & Method 2 \\
(IWT-DPCM) & (DPCM-IWT) \\
\hline Lena & 4.8954 & 4.7555 \\
Baboon & 6.4361 & 6.3424 \\
Goldhill & 5.0319 & 5.5163 \\
Peppers & 5.0009 & 4.9787 \\
Cameraman & 3.7192 & 3.5825 \\
\hline
\end{tabular}

The criterion used to compare the performance is the ratio of the quantity of size reduction (with respect to the number of bytes) of thle original file, given by; 
Table 2 shows the comparison of compression ratio resulted after the image has been encoded by Huffman. Some established methods using bit reduction to show their result. Since, many of them using compression ratio unit, so we use it as a standard comparision. It shows that most of the images give high compression ratio by applying method 2 compared to method 1 .

Table 2. Compression ratio comparison

\begin{tabular}{|c|c|c|}
\hline \multirow[b]{2}{*}{ Images } & \multicolumn{2}{|c|}{ Predictive method } \\
\hline & $\begin{array}{c}\text { Method } 1 \\
\text { (IWT-DPCM) }\end{array}$ & $\begin{array}{c}\text { Method } 2 \\
\text { (DPCM-IWT) }\end{array}$ \\
\hline Lena & 1.4999 & 1.5457 \\
\hline Baboon & 1.0258 & 1.0522 \\
\hline Goldhill & 1.3181 & 1.2576 \\
\hline Peppers & 1.4471 & 1.4644 \\
\hline Cameraman & 2.0469 & 2.1214 \\
\hline
\end{tabular}

The perfect reconstruction of images is clarified by displaying the original picture in Figure 7 with the picture that has been decoded in Figure 8. The difference of the reconstructed image and the original image will give black image since all the pixels will become 0 as there is no error in the reconstructed image.

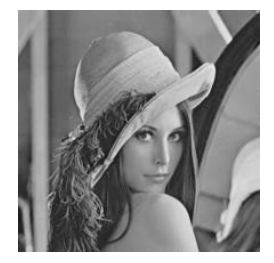

Figure 7. Original image

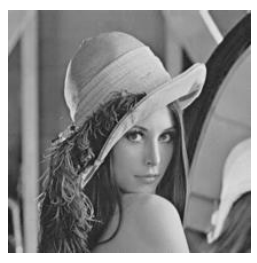

Figure 8. Reconstruct image

The performance of hybrid predictive between the proposed methods has been compared by calculating the entropy value and compression ratio. From the analysis, it can be concluded that DPCM-IWT is a better hybrid predictive method as it produces lower entropy value and compression ratio. The number of bits of Lena is reduced by $36 \%$, Cameraman is reduced by $48 \%$, Peppers is reduced by $34 \%$ and Baboon is reduced by $13 \%$ after applying hybrid predictive of DPCM-IWT. The truncated Huffman table that has been designed is applicable to be used for the tested image as most of the image is having the same cut-off pixel distribution and the design of Huffman truncated table is flexible to be used in any image as the codeword could be assigned in any symbol manually. By implementing hybrid predictive of DPCM-IWT through the lossless image compression, it contributes to optimized performance of lossless image compression field.

\section{CONCLUSION}

It is found that the combination of predictive DPCM and IWT could improve the performance of predictive coding of lossless image compression. The resulted analysis found that to achieve maximum compression of hybrid predictive coding, the resulted approximation sub-band that is encoded by IWT needs to be encoded with DPCM. From the analysis, the hybrid predictive technique with sequence DPCM-IWT-Huffman performs better with lower entropy and compression ratio. The perfect reconstruction of the image is clarified by comparing the difference between constructed image and the original image. The resulted black image of the difference has proven that there is no loss for the predicted pixel. In summary, the proposed hybrid predictive technique for lossless image compression is a promising solution to achieve faster transmission speed and lower storage space for image transmission over telecommunication network for applications such as healthcare network and biometric security solution. 


\section{ACKNOWLEDGEMENTS}

The author would like to thank Universiti Teknologi Malaysia and Universiti Tun Hussein Onn Malaysia. This work has been supported by Universiti Tun Hussein Onn Malaysia under Short Term Grant (STG) Vote U655.

\section{REFERENCES}

[1] Barni, M., "Document and Image Compression," Boca Raton, Fla: Taylor \& Francis, 2006.

[2] Hu, Y.-C. and C.-C. Chang, "A new lossless compression scheme based on Huffman coding scheme for image compression,” Signal Processing: Image Communication, 16(4): p. 367-372, 2000.

[3] Kavitha, P., "A Survey on Lossless and Lossy Data Compression Methods," International Journal of Computer Science \& Engineering Technology (IJCSET), 7(03), 2016.

[4] Min Shi and Shengli Xie, "A lossless image compression algorithm by combining DPCM with integer wavelet transform," Proceedings of the IEEE 6th Circuits and Systems Symposium on Emerging Technologies: Frontiers of Mobile and Wireless Communication (IEEE Cat. No.04EX710), Shanghai, China, 2004, pp. 293-296 Vol.1.

[5] Meyer, B. and P. Tischer, "TMW-a new method for lossless image compression," ITG Fachbericht, p.533-540, 1997.

[6] A. Lempel and J. Ziv, "On the complexity of finite sequences,"in IEEE Trans. Inform. Theory, vol. 22, no. 1, pp. 75-81, January 1976.

[7] J. Zhang, and G. Liu, "A novel lossless compression for hyperspectral images by context-based adaptive classified arithmetic coding in wavelet domain," IEEE Geosci. Remote Sens. Lett, vol. 4, no. 3, July, 2007.

[8] J. Zhang, and G. Liu. "A novel lossless compression for hyperspectral images by adaptive classified arithmetic coding in wavelet domain," In Proc. ICIP, pp. 2269-2272, Oct. 2006.

[9] E. Magli, G. Olmo, and E. Quacchio. "Optimized onboard lossless and near-lossless compression of hyperspectral data using CALIC," IEEE Geosci. Remote Sens. Lett, vol. 1, no. 1, pp. 21-25, Jan. 2004.

[10] Y. Wang, "A set of transforms for lossless image compression,"in IEEE Transactions on Image Processing 4 (5), 1995.

[11] N. Ranganathan, S. Romaniuk, K.R. Namuduri, "A lossless image compression algorithm using variable block size segmentation," in IEEE Transactions on Image Processing 4 (10), 1995.

[12] En-Hui Yang; Yunwei Jia, "Universal lossless coding of sources with large and unbounded alphabets," in IEEE International Symposium on Information Theory, page 16, 2000.

[13] J. G. Cleary and I. H. Witten. "Data compression using adaptive coding and partial string matching," in IEEE Transactions on Communications, 32(4):396-402, 1984.

[14] J. J. Rissanen. "Universal coding, information, prediction and estimation," in IEEE Transactions on Information Theory, vol. 30, no. 4, pp. 629-636, July 1984.

[15] L. D. Davisson, "Universal noiseless coding," IEEE Trans. Inform. Theory, vol. IT-19, pp. 783-795, Nov. 1973.

[16] Liang Shen, Rangaraj and M. Rangayyan, "A Segmentation-Based Lossless Image Coding Method for High-Resolution Medical Image Compression,” in IEEE Trans. Medical Image, vol. 16, no. 3, pp. 301-307, 1997

[17] Jiebo Luo, Xiaohui Wang, Chang Wen Chen and Kevin J.Parker, "Volumetric Medical Image Compression with Three dimensional Wavelet Transform and Octave Zerotree Coding," Proceedings of SPIE, vol. 2727, pp. 579-590, 1996.

[18] G.K.Wallace, "The JPEG still picture compression standard," Communications of the ACM, Vol. 34, pp. 30-44, 1991.

[19] D. Santa-Cruza, T. Ebrahimia, J. Askelöfb, M. Larssonb and C. A. Christopoulosb, "JPEG 2000 still image coding versus other standards," Proceedings of SPIE's 45th annual meeting, Applications of Digital Image Processing XXIII, vol. 4115, pp. 446-454, 2000.

[20] Jan-Yie Liang, Chih-Sheng Chen, Chua-Huang Huang and Li Liu, "Lossless compression of medical images using Hilbert space-filling curves," Computerized Medical Imaging and Graphics, vol. 32, Issue 3, pp.174-182, 2008.

[21] Yong Zhang, Donald A. Adjeroh, "Prediction by Partial Approximate Matching for Lossless Image Compression," in IEEE Trans. Image Processing, vol. 17, no. 6, pp. 924-935, June 2008.

[22] Mofreh, A., T.M. Barakat, and A.M. Refaat, "A New Lossless Medical Image Compression Technique using Hybrid Prediction Model,” Signal Processing: An International Journal (SPIJ), 10(3): p. 20, 2016.

[23] C. Jain, V. Chaudhary, K. Jain and S. Karsoliya, "Performance analysis of integer wavelet transform for image compression," 2011 3rd International Conference on Electronics Computer Technology, Kanyakumari, 2011, pp. 244-246.

[24] Singh, M., S. Kumar, and S. Singh, "Various Image Compression Techniques: Lossy and Lossless," Image, 142(6), 2016.

[25] Gupta, V., A. Rao, and K.M. Pandey, "Integer Wavelet Transform and Predictive Coding Technique for Lossless Medical Image Compression,” International Journal of Technology Exploration and learning, 5, 2013. 


\section{BIOGRAPHIES OF AUTHORS}
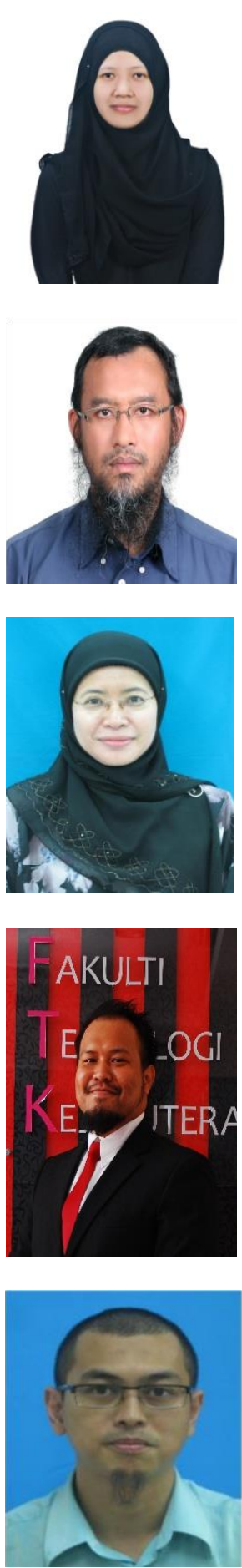

Nur Aqilah Nor Azman received her Degree in Electrical-Electronic Engineering from Universiti Teknologi Malaysia, Skudai, Johor, Malaysia, in 2018. Currently, she is working as a Soc Design Engineer at Intel Malaysia.

Samura Ali received his MSc in Real-time Communication and Electronic System from University of Bradford, UK in 1998. Currently, he is a senior lecturer at the School of Electrical Engineering, Universiti Teknologi Malaysia, Malaysia. His current research interests include image encryption and compression.

Rozeha A. Rashid received her B.Sc. degree in electrical engineering from the University of Michigan, Ann Arbor, USA and her M.E.E. and PhD degrees in telecommunication engineering from the Universiti Teknologi Malaysia (UTM). She is a senior lecturer in the Communication Engineering Program, School of Electrical Engineering, Universiti Teknologi Malaysia and currently is the Head of Advanced Telecommunication Technology (ATT) research group. Her current research interests include wireless communications, sensor network, cognitive radio and Internet-of-Things.

Faiz Asraf Saparudin received his Bachelor B.Sc. in Electrical Engineering (Telecommunication) with First Class Honours from Universiti Teknologi Malaysia in 2010 and received WAMY Academic Excellence Award in the same year. Ph.D. degree in Electrical Engineering (Telecommunication) from the Universiti Teknologi Malaysia in 2015. He is currently a Faculty Member in Fakulti Teknologi Kejuruteraan, Universiti Tun Hussein Onn Malaysia. Member of Institue of Electrical and Electronic Engineers (IEEE) and IEEE Communication Society (ComSoc). His current research interests include radio resource management, distributed algorithms, nature-inspired techniques, multiagent system and game theoretic approach for next-generation mobile network.

Mohd Adib Sarijari received his Bachelors in Engineering degree (first class, and with honors) in 2007, and the Master of Science in Electrical Engineering degree in 2011, both from Universiti Teknologi Malaysia (UTM), Johor, Malaysia. In 2016, he received his PhD from Delft University of Technology (TU Delft), the Netherlands under the Circuit and System Group (Signal Processing for Communication section). He is currently a Senior Lecturer at the Department of Communication Engineering at the Faculty of Electrical Engineering, UTM. His general research interest lies in the field of communications, optimization, and system design. In particular, he is interested in Cognitive Radio, Home Area Networks, Wireless Sensor Networks, Internet-of-Things, Software Defined Radio, and Smart City. 